\title{
Kohn-Vogelius formulation and high-order topological asymptotic formula for identifying small obstacles in a fluid medium
}

\author{
Montassar Barhoumi
}

\begin{abstract}
This paper concerns the identification of a small obstacle immersed in a Stokes flow from boundary measurements. The proposed approach is based on the Kohn-Vogelius formulation and the topological sensitivity analysis method. We derive a high order asymptotic formula describing the variation of a Kohn-Vogelius type functional with respect to the insertion of a small obstacle inside the fluid flow domain. The obtained asymptotic formula will serve as very useful tools for developing accurate and robust numerical reconstruction algorithms.
\end{abstract}

\section{Introduction}

The development and application of the nondestructive inspection technique has recently received considerable interest in the engineering as well as in the applied mathematics. Such a technique leads to determine interior information about medium from boundary measurements data.

For this purpose, several approaches and methods have been developed, one can cite: the identification of cracks by boundary measurements $[1,2,3,4,5]$, the reconstruction of electromagnetic imperfections of a small diameter from measurements on the boundary $[6,7]$, the determination of small conductivity imperfections inside a conductor have been established in $[8,9,10]$, the

Key Words: Kohn-Vogelius formulation, Topological sensitivity analysis, Stokes system, Geometric inverse problem, Calculus of variations, Asymptotic formula.

2010 Mathematics Subject Classification: Primary 35N25; Secondary 49K40.

Received: 19.02.2019.

Accepted: 25.04 .2019 . 
KOHN-VOGELIUS FORMULATION AND HIGH-ORDER TOPOLOGICAL ASYMPTOTIC FORMULA FOR IDENTIFYING SMALL OBSTACLES IN A FLUID MEDIUM

reconstruction of imperfections of small diameter in an elastic medium using boundary integral formula $[11,12]$, the identification of a finite number of interior particles of small diameter related to the full Maxwell equations [13], ... etc.

This paper is concerned with the fluid mechanics area. The objective of this work is to derive a high-order asymptotic formula leading to determine the location, shape and size of an obstacle of a small volume immersed in a fluid medium from boundary measurements.

The proposed method is based on the Kohn-Vogelius formulation [14, 15] and the topological sensitivity analysis method [16, 17, 18, 19, 20, 21, 22, 23]. We derive a high-order topological asymptotic expansion for a Kohn-Vogelius type functional with respect to the presence of a small obstacle inside the fluid flow domain. An asymptotic formula is derived giving the relation between the known boundary data and the unknown obstacle properties; location, size and shape.

The obtained asymptotic formula will serve as very useful tools for developing very effective reconstruction algorithms for identifying small obstacles from boundary measurements. Such algorithms can be applied in various applications like fiber-reinforced polymers [24, 25], colloid [26, 27, 28] and casting or injection filling $[29,30,31]$ where the design of the mixing of liquid metallic should be optimized.

The rest of this paper is organized as follows. In Section 2, we present the formulation of the considered problem. We introduce the Kohn-Vogelius concept and the topological sensitivity technique. The obstacle reconstruction problem is formulated as a topology optimization one minimizing a KohnVogelius type functional. Section 3 is devoted to a preliminary estimate describing the variation of the considered Kohn-Vogelius functional with respect to the presence of a small obstacle. In Section 4, we establish an asymptotic expansion for the perturbed velocity and pressure fields. In Section 5, we extend the topological derivative notion for the high-order case. We derive a high-order topological asymptotic formula for the Stokes operator. The paper ends by a conclusion and perspective works.

\section{Formulation of the problem}

We consider a viscous and incompressible fluid flow in a bounded domain $\Omega$ of $\mathbb{R}^{3}$, with smooth boundary $\partial \Omega$. Let $\Gamma$ and $\Sigma$ be two parts of $\partial \Omega$ such that $\overline{\partial \Omega}=\bar{\Gamma} \cup \bar{\Sigma}$ and $\Gamma \cap \Sigma=\emptyset$. We assume that the fluid flow is in a laminar regime, in such way that the convection term can be neglected and the NavierStokes equations may be approximated by the non-stationary Stokes system. 
Inside the fluid flow domain, we assume the existence of a small obstacle $\mathcal{O}_{z, \varepsilon}=z+\varepsilon \mathcal{O}$ that is characterized by its center $z$, its size $\varepsilon$ and its shape $\mathcal{O}$, with $\mathcal{O}$ is a bounded domain of $\mathbb{R}^{3}$ containing the origin, whose boundary $\partial \mathcal{O}$ is connected and piecewise of class $\mathrm{e}^{1}$.

The aim of this work is to develop an efficient approach for identifying the unknown parameters (location, size and shape) of the obstacle $\mathcal{O}_{z, \varepsilon}$ from boundary measurement of the velocity field on a part of the boundary. This issue can be formulated as a geometric inverse problem as follow:

- Given a Neumann and Dirichlet data on the accessible boundary $\Sigma$ : an imposed force $G$ and a measured velocity field $\mathcal{W}_{m}$.

- Determine the location $z$, size $\varepsilon$ and shape $\mathcal{O}$ of the unknown obstacle $\mathcal{O}_{z, \varepsilon}$ such that the solution $\left(w_{\mathcal{O}_{z, \varepsilon}}, q_{\mathcal{O}_{z, \varepsilon}}\right)$ of the Stokes equations satisfies the following over-determined boundary value problem

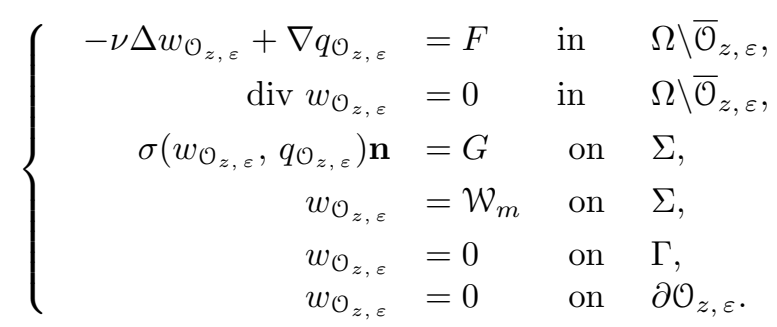

where $w_{\mathcal{O}_{z, \varepsilon}}$ is the velocity field, $q_{\mathcal{O}_{z, \varepsilon}}$ is the pressure field, $\nu$ is the kinematic viscosity coefficient of the fluid and $F$ is the gravitational force.

In order to examine the considered geometric inverse problem, we propose in this paper a new approach combining the advantages of the Kohn-Vogelius formulation and the topological sensitivity analysis method.

\subsection{Kohn-Vogelius formulation}

The first step of our approach is based on the Kohn-Vogelius formulation which rephrase the considered geometric inverse problem into a topology optimization one. It leads to define for any given obstacle $\mathcal{O}_{z, \varepsilon}$ two forward problems: The first one which be called Neumann problem is associated to the Neumann datum $F$ :

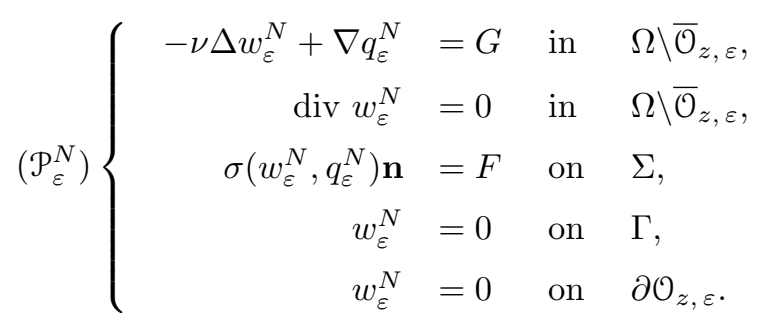


The second one is associated to the measured velocity $\mathcal{W}_{m}$ which will be called as the Dirichlet problem:

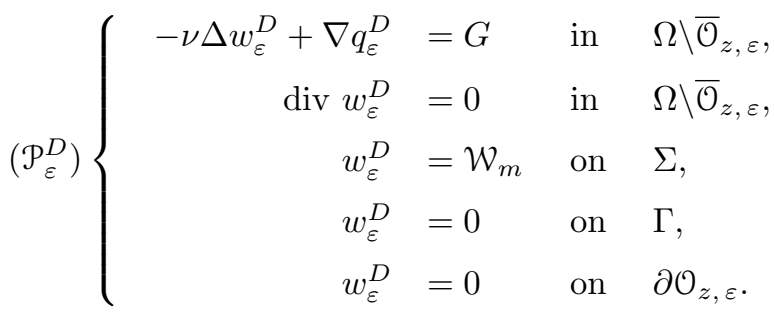

As one can remark here, if $\mathcal{O}_{z, \varepsilon}$ coincides with the exact obstacle $\mathcal{O}_{z^{*}, \varepsilon^{*}}=$ $z^{*}+\varepsilon^{*} \mathcal{O}^{*}$, the misfit between the solutions become zero $\left(w_{\varepsilon}^{N}=w_{\varepsilon}^{D}\right)$. Starting from this remark, the inverse problem can be formulated as a topology optimization one. The unknown obstacle will be characterized as the minimum of the following Kohn-Vogelius type functional [15]

$$
\mathcal{K}\left(\Omega \backslash \overline{\mathcal{O}}_{z, \varepsilon}\right)=\nu \int_{\Omega \backslash \overline{\mathcal{O}}_{z, \varepsilon}}\left|\nabla w_{\varepsilon}^{N}-\nabla w_{\varepsilon}^{D}\right|^{2} d x .
$$

More precisely, the identification problem can be formulated as follow:

$$
\left(\mathcal{P}_{\text {opt }}\right)\left\{\begin{array}{l}
\text { find } \mathcal{O}_{z^{*}, \varepsilon^{*}} \subset \Omega, \text { such that } \\
\mathcal{K}\left(\Omega \backslash \overline{\mathcal{O}}_{z^{*}, \varepsilon^{*}}\right)=\min _{\mathcal{O}_{z, \varepsilon} \in \mathcal{D}_{a d}} \mathcal{K}\left(\Omega \backslash \overline{\mathcal{O}}_{z, \varepsilon}\right),
\end{array}\right.
$$

where $\mathcal{D}_{a d}$ is a given set of admissible domains.

It is interesting to note that, in the absence of obstacles the function $\mathcal{K}$ reads

$$
\mathcal{K}(\Omega)=\nu \int_{\Omega}\left|\nabla w_{0}^{N}-\nabla w_{0}^{D}\right|^{2} d x,
$$

where $\left(w_{0}^{N}, q_{0}^{N}\right)$ and $\left(w_{0}^{D}, q_{0}^{D}\right)$ satisfy the Stokes equations in the non perturbed domain

$$
\begin{array}{r}
\left(\mathcal{P}_{0}^{N}\right)\left\{\begin{array}{rlll}
-\nu \Delta w_{0}^{N}+\nabla q_{0}^{N} & =G & \text { in } & \Omega, \\
\operatorname{div} w_{0}^{N} & =0 & \text { in } & \Omega, \\
\sigma\left(w_{0}^{N}, q_{0}^{N}\right) \mathbf{n} & =F & \text { on } & \Sigma, \\
w_{\varepsilon}^{N} & =0 & \text { on } & \Gamma,
\end{array}\right. \\
\left(\mathcal{P}_{0}^{D}\right)\left\{\begin{array}{rlll}
-\nu \Delta w_{0}^{D}+\nabla q_{0}^{D} & =G & \text { in } & \Omega, \\
\operatorname{div} w_{0}^{D} & =0 & \text { in } & \Omega, \\
w_{0}^{D} & =\mathcal{W}_{m} & \text { on } & \Sigma, \\
w_{0}^{D} & =0 & \text { on } & \Gamma .
\end{array}\right.
\end{array}
$$


KOHN-VOGELIUS FORMULATION AND HIGH-ORDER TOPOLOGICAL ASYMPTOTIC FORMULA FOR IDENTIFYING SMALL OBSTACLES IN A FLUID MEDIUM

\subsection{Sensitivity analysis method}

To solve the topological optimization problem $\left(\mathcal{P}_{\text {opt }}\right)$ and identify the unknown obstacle, we will propose a simplified approach based on the topological sensitivity analysis for the Kohn-Vogelius function $\mathcal{K}$.

The classical topological sensitivity analysis method is based on a first order asymptotic expansion of the form

$$
\mathcal{K}\left(\Omega \backslash \overline{\mathcal{O}}_{z, \varepsilon}\right)-\mathcal{K}(\Omega)=\rho(\varepsilon) \delta \mathcal{K}(z)+o(\rho(\varepsilon)), \forall z \in \Omega,
$$

where

- $\varepsilon \mapsto \rho(\varepsilon)$ is a positive scalar function going to zero with $\varepsilon$.

. $z \mapsto \delta \mathcal{K}(z)$ is the first order topological gradient.

In the context of fluid mechanics, the first order topological sensitivity analysis has been derived for the Stokes operator in [19, 21, 32], the quasi-Stokes in [22] and for the stationary Navier-Stokes equations in [20]. In [33], Caubet and Dambrine have used the Kohn-Vogelius formulation combined with the topological sensitivity analysis to locate obstacles in a fluid flow domain.

In this work, we extend the topological sensitivity notion for the high-order case. We will derive a high-order topological asymptotic expansion for the Kohn-Vogelius functional $\mathcal{K}$ with respect to the presence of the Dirichlet geometric perturbation $\mathcal{O}_{z, \varepsilon}$ inside the domain $\Omega$. It consists in studying the variation $\mathcal{K}\left(\Omega \backslash \overline{\mathcal{O}}_{z, \varepsilon}\right)-\mathcal{K}(\Omega)$ with respect to $\varepsilon$ and establishing an asymptotic formula on the form

$$
\mathcal{K}\left(\Omega \backslash \overline{\mathcal{O}}_{z, \varepsilon}\right)-\mathcal{K}(\Omega)=\sum_{q=1}^{m} \rho_{q}(\varepsilon) \delta \mathcal{K}^{q}(z, \mathcal{O})+o\left(\rho_{m}(\varepsilon)\right)
$$

where

- $\varepsilon \mapsto \rho_{q}(\varepsilon), 1 \leq q \leq m$ are positive scalar functions defined on $\mathbb{R}^{+}$ verifying $\rho_{q+1}(\varepsilon)=o\left(\rho_{q}(\varepsilon)\right)$ and $\lim _{\varepsilon \rightarrow 0} \rho_{q}(\varepsilon)=0$.

- $\delta \mathcal{K}^{q}$ denotes the $q^{\text {th }}$ topological derivative of the functional $\mathcal{K}$.

- $m \in \mathbb{N}^{*}$ an arbitrary order of the asymptotic expansion.

To this end, we start our analysis by deriving a preliminary result showing the relationship between the quantity $\mathcal{K}\left(\Omega \backslash \overline{\mathcal{O}}_{z, \varepsilon}\right)-\mathcal{K}(\Omega)$ and the variations of the Neumann and Dirichlet perturbed solutions $\left(w_{\varepsilon}^{N}-w_{0}^{N}, q_{\varepsilon}^{N}-q_{0}^{N}\right)$ and $\left(w_{\varepsilon}^{D}-w_{0}^{D}, q_{\varepsilon}^{D}-q_{0}^{D}\right)$. 
KOHN-VOGELIUS FORMULATION AND HIGH-ORDER TOPOLOGICAL ASYMPTOTIC FORMULA FOR IDENTIFYING SMALL OBSTACLES IN A FLUID MEDIUM

\section{The Kohn-Vogelius functional variation}

In this section, we discuss the sensitivity of the considered Kohn-Vogelius functional $\mathcal{K}$ with respect to the presence of a small obstacle $\mathcal{O}_{z, \varepsilon}$ inside the fluid flow domain. We will prove that the variation $\mathcal{K}\left(\Omega \backslash \overline{\mathcal{O}}_{z, \varepsilon}\right)-\mathcal{K}(\Omega)$ can be only described by four integral terms, involving the discrepancy between the initial and the perturbed Stokes problem solutions.

Theorem 3.1. Let $\mathcal{O}_{z, \varepsilon}$ be a small obstacle, strictly embedded into the fluid flow domain $\Omega$. In the presence of $\mathcal{O}_{z, \varepsilon}$, the Kohn-Vogelius function $\mathcal{K}$ admits the following variation

$$
\begin{aligned}
\mathcal{K}\left(\Omega \backslash \overline{\mathcal{O}}_{z, \varepsilon}\right)-\mathcal{K}(\Omega)= & \int_{\partial \mathcal{O}_{z, \varepsilon}} \sigma\left(w_{\varepsilon}^{N}-w_{0}^{N}, q_{\varepsilon}^{N}-q_{0}^{N}\right) \mathbf{n} w_{0}^{N} d s \\
& -\int_{\partial \mathcal{O}_{z, \varepsilon}} \sigma\left(w_{\varepsilon}^{D}-w_{0}^{D}, q_{\varepsilon}^{D}-q_{0}^{D}\right) \mathbf{n} w_{0}^{D} d s \\
& +\nu \int_{\mathcal{O}_{z, \varepsilon}}\left|\nabla w_{0}^{D}\right|^{2} d x-\nu \int_{\mathcal{O}_{z, \varepsilon}}\left|\nabla w_{0}^{N}\right|^{2} d x .
\end{aligned}
$$

Proof. The variation of the function $\mathcal{K}$ is given by

$$
\begin{aligned}
\mathcal{K}\left(\Omega \backslash \overline{\mathcal{O}}_{z, \varepsilon}\right)-\mathcal{K}(\Omega) & =\nu \int_{\Omega \backslash \overline{\mathcal{O}}_{z, \varepsilon}}\left|\nabla w_{\varepsilon}^{N}-\nabla w_{\varepsilon}^{D}\right|^{2} d x-\nu \int_{\Omega}\left|\nabla w_{0}^{N}-\nabla w_{0}^{D}\right|^{2} d x \\
= & \nu \int_{\Omega \backslash \overline{\mathcal{O}}_{z, \varepsilon}}\left|\nabla w_{\varepsilon}^{N}\right|^{2} d x+\nu \int_{\Omega \backslash \overline{\mathcal{O}}_{z, \varepsilon}}\left|\nabla w_{\varepsilon}^{D}\right|^{2} d x-2 \nu \int_{\Omega \backslash \overline{\mathcal{O}}_{z, \varepsilon}} \nabla w_{\varepsilon}^{D} \nabla w_{\varepsilon}^{N} d x \\
& -\nu \int_{\Omega}\left|\nabla w_{0}^{N}\right|^{2} d x-\nu \int_{\Omega}\left|\nabla w_{0}^{D}\right|^{2} d x+2 \nu \int_{\Omega \backslash \overline{\mathcal{O}}_{z, \varepsilon}} \nabla w_{0}^{D} \nabla w_{0}^{N} d x .
\end{aligned}
$$

As one can observe $\mathcal{K}\left(\Omega \backslash \overline{\mathcal{O}}_{z, \varepsilon}\right)-\mathcal{K}(\Omega)$ can be decomposed as

$$
\mathcal{K}\left(\Omega \backslash \overline{\mathcal{O}}_{z, \varepsilon}\right)-\mathcal{K}(\Omega)=\mathcal{T}_{N}(\varepsilon)+\mathcal{T}_{D}(\varepsilon)-2 \mathcal{T}_{M}(\varepsilon),
$$

with $\mathcal{T}_{N}$ is the Neumann term

$$
\mathcal{T}_{N}(\varepsilon)=\nu \int_{\Omega \backslash \overline{\mathcal{O}}_{z, \varepsilon}}\left|\nabla w_{\varepsilon}^{N}\right|^{2} d x-\nu \int_{\Omega}\left|\nabla w_{0}^{N}\right|^{2} d x,
$$

$\mathcal{T}_{D}$ is the Dirichlet term

$$
\mathcal{T}_{D}(\varepsilon)=\nu \int_{\Omega \backslash \overline{\mathcal{O}}_{z, \varepsilon}}\left|\nabla w_{\varepsilon}^{D}\right|^{2} d x-\nu \int_{\Omega}\left|\nabla w_{0}^{D}\right|^{2} d x,
$$

and $\mathcal{T}_{M}$ is the mixed term

$$
\mathcal{T}_{M}(\varepsilon)=\nu \int_{\Omega \backslash \overline{\mathcal{O}}_{z, \varepsilon}} \nabla w_{\varepsilon}^{D} \nabla w_{\varepsilon}^{N} d x-\nu \int_{\Omega \backslash \overline{\mathcal{O}}_{z, \varepsilon}} \nabla w_{0}^{D} \nabla w_{0}^{N} d x .
$$


KOHN-VOGELIUS FORMULATION AND HIGH-ORDER TOPOLOGICAL ASYMPTOTIC FORMULA FOR IDENTIFYING SMALL OBSTACLES IN A FLUID MEDIUM

Next, we will examine each term separately. We start our analysis by studying the term $\mathcal{T}_{N}$.

- Variation of the Neumann term: The term $\mathcal{T}_{N}$ reads

$$
\begin{aligned}
\mathcal{T}_{N}(\varepsilon) & =\nu \int_{\Omega \backslash \overline{\mathcal{O}}_{z, \varepsilon}}\left|\nabla w_{\varepsilon}^{N}\right|^{2} d x-\nu \int_{\Omega}\left|\nabla w_{0}^{N}\right|^{2} d x \\
& =\nu \int_{\Omega \backslash \overline{\mathcal{O}}_{z, \varepsilon}} \nabla\left(w_{\varepsilon}^{N}-w_{0}^{N}\right) \nabla w_{\varepsilon}^{N} d x+\nu \int_{\Omega \backslash \overline{\mathcal{O}}_{z, \varepsilon}} \nabla\left(w_{\varepsilon}^{N}-w_{0}^{N}\right) \nabla w_{0}^{N} d x \\
& -\nu \int_{\mathcal{O}_{z, \varepsilon}}\left|\nabla w_{0}^{N}\right|^{2} d x .
\end{aligned}
$$

Using Green formula, from the Stokes system satisfied by $\left(w_{\varepsilon}^{N}-w_{0}^{N}, q_{\varepsilon}^{N}-q_{0}^{N}\right)$, we obtain

$$
\nu \int_{\Omega \backslash \overline{\mathcal{O}}_{z, \varepsilon}} \nabla\left(w_{\varepsilon}^{N}-w_{0}^{N}\right) \nabla w_{0}^{N} d x=\int_{\partial \mathcal{O}_{z, \varepsilon}} \sigma\left(w_{\varepsilon}^{N}-w_{0}^{N}, q_{\varepsilon}^{N}-q_{0}^{N}\right) \mathbf{n} w_{0}^{N} d s .
$$

In addition,

$$
\nu \int_{\Omega \backslash \overline{\mathcal{O}}_{z, \varepsilon}} \nabla\left(w_{\varepsilon}^{N}-w_{0}^{N}\right) \nabla w_{\varepsilon}^{N} d x d t=0
$$

Then, the term $\mathcal{T}_{N}$ can be written as

$$
\mathcal{T}_{N}(\varepsilon)=\int_{\partial \mathcal{O}_{z, \varepsilon}} \sigma\left(w_{\varepsilon}^{N}-w_{0}^{N}, q_{\varepsilon}^{N}-q_{0}^{N}\right) \mathbf{n} w_{0}^{N} d s-\nu \int_{\mathcal{O}_{z, \varepsilon}}\left|\nabla w_{0}^{N}\right|^{2} d x
$$

- Variation of the Dirichlet term: We have

$$
\begin{aligned}
& \mathcal{T}_{D}(\varepsilon)=\nu \int_{\Omega \backslash \overline{\mathrm{O}}_{z, \varepsilon}}\left|\nabla w_{\varepsilon}^{D}\right|^{2} d x-\nu \int_{\Omega}\left|\nabla w_{0}^{D}\right|^{2} d x \\
& =\nu \int_{\Omega \backslash \overline{\mathcal{O}} z, \varepsilon}\left|\nabla\left(w_{\varepsilon}^{D}-w_{0}^{D}\right)\right|^{2} d x+2 \nu \int_{\Omega \backslash \overline{\mathcal{O}}_{z, \varepsilon}} \nabla\left(w_{\varepsilon}^{D}-w_{0}^{D}\right) \nabla w_{0}^{D} d x-\nu \int_{\mathcal{O}_{z, \varepsilon}}\left|\nabla w_{0}^{D}\right|^{2} d x .
\end{aligned}
$$

Using Green formula, the system $\left(\mathcal{P}_{0}^{D}\right)$ implies

$\nu \int_{\Omega \backslash \overline{\mathcal{O}}_{z, \varepsilon}} \nabla\left(w_{\varepsilon}^{D}-w_{0}^{D}\right) \nabla w_{0}^{D} d x=\int_{\Omega \backslash \overline{\mathcal{O}}_{z, \varepsilon}} F\left(w_{\varepsilon}^{D}-w_{0}^{D}\right) d x-\int_{\partial \mathcal{O}_{z, \varepsilon}} \sigma\left(w_{0}^{D}, q_{0}^{D}\right) \mathbf{n} w_{0}^{D} d s$.

Then, the Dirichlet term admits the following variation

$$
\begin{aligned}
\mathcal{T}_{D}(\varepsilon)= & \nu \int_{\Omega \backslash \overline{\mathcal{O}}_{z, \varepsilon}}\left|\nabla\left(w_{\varepsilon}^{D}-w_{0}^{D}\right)\right|^{2} d x+2 \int_{\Omega \backslash \overline{\mathcal{O}}_{z, \varepsilon}} F\left(w_{\varepsilon}^{D}-w_{0}^{D}\right) d x \\
& -\nu \int_{\mathcal{O}_{z, \varepsilon}}\left|\nabla w_{0}^{D}\right|^{2} d x-2 \int_{\partial \mathcal{O}_{z, \varepsilon}} \sigma\left(w_{0}^{D}, q_{0}^{D}\right) \mathbf{n} w_{0}^{D} d s .
\end{aligned}
$$


Using Green formula, from the system verified by $\left(w_{\varepsilon}^{D}-w_{0}^{D}, q_{\varepsilon}^{D}-q_{0}^{D}\right)$, we deduce

$$
\begin{aligned}
\mathcal{T}_{D}(\varepsilon)= & -\int_{\partial \mathcal{O}_{z, \varepsilon}} \sigma\left(w_{\varepsilon}^{D}-w_{0}^{D}, q_{\varepsilon}^{D}-q_{0}^{D}\right) \mathbf{n} w_{0}^{D} d s+2 \int_{\Omega \backslash \overline{\mathcal{O}}_{z, \varepsilon}} F\left(w_{\varepsilon}^{D}-w_{0}^{D}\right) d x \\
& -2 \int_{\partial \mathcal{O}_{z, \varepsilon}} \sigma\left(w_{0}^{D}, q_{0}^{D}\right) \mathbf{n} w_{0}^{D} d s-\nu \int_{\mathcal{O}_{z, \varepsilon}}\left|\nabla w_{0}^{D}\right|^{2} d x
\end{aligned}
$$

- Variation of the mixed term: We have

$$
\mathcal{T}_{M}(\varepsilon)=\nu \int_{\Omega \backslash \overline{\mathcal{O}}_{z, \varepsilon}} \nabla w_{\varepsilon}^{N} \nabla w_{\varepsilon}^{D} d x d t-\nu \int_{\Omega} \nabla w_{0}^{N} \nabla w_{0}^{D} d x
$$

From the weak formulation of problems $\left(\mathcal{P}_{\varepsilon}^{N}\right)$ and $\left(\mathcal{P}_{0}^{N}\right)$, it follows that

$$
\mathcal{T}_{M}(\varepsilon)=\int_{\Omega \backslash \overline{\mathcal{O}}_{z, \varepsilon}} F\left(w_{\varepsilon}^{D}-w_{0}^{D}\right) d x-\int_{\mathcal{O}_{z, \varepsilon}} F w_{0}^{D} d x .
$$

From the fact that $-\nu \Delta w_{0}^{D}+\nabla q_{0}^{D}=F$ in $\mathcal{O}_{z, \varepsilon}$ and $\operatorname{div} w_{0}^{D}=0$ in $\mathcal{O}_{z, \varepsilon}$, one can deduce

$$
\int_{\mathcal{O}_{z, \varepsilon}} F w_{0}^{D} d x=\nu \int_{\mathcal{O}_{z, \varepsilon}}\left|\nabla w_{0}^{D}\right|^{2} d x+\int_{\partial \mathcal{O}_{z, \varepsilon}} \sigma\left(w_{0}^{D}, q_{0}^{D}\right) \mathbf{n} w_{0}^{D} d s .
$$

Consequently, the term $\mathcal{T}_{M}$ can be written as

$$
\mathcal{T}_{M}(\varepsilon)=\int_{\Omega \backslash \overline{\mathcal{O}}_{z, \varepsilon}} F\left(w_{\varepsilon}^{D}-w_{0}^{D}\right) d x-\nu \int_{\mathcal{O}_{z, \varepsilon}}\left|\nabla w_{0}^{D}\right|^{2} d x-\int_{\partial \mathcal{O}_{z, \varepsilon}} \sigma\left(w_{0}^{D}, q_{0}^{D}\right) \mathbf{n} w_{0}^{D} d s .
$$

- Variation of the Kohn-Vogelius function $\mathcal{K}$ : Combining the variations (3), (4) and (5), one can deduce that the variation of $\mathcal{K}$ can be written as

$$
\begin{aligned}
& \mathcal{K}\left(\Omega \backslash \overline{\mathcal{O}}_{z, \varepsilon}\right)-\mathcal{K}(\Omega)=\int_{\partial \mathcal{O}_{z, \varepsilon}} \sigma\left(w_{\varepsilon}^{N}-w_{0}^{N}, q_{\varepsilon}^{N}-q_{0}^{N}\right) \mathbf{n} w_{0}^{N} d s-\nu \int_{\mathcal{O}_{z, \varepsilon}}\left|\nabla w_{0}^{N}\right|^{2} d x \\
&+\nu \int_{\mathcal{O}_{z, \varepsilon}}\left|\nabla w_{0}^{D}\right|^{2} d x-\int_{\partial \mathcal{O}_{z, \varepsilon}} \sigma\left(w_{\varepsilon}^{D}-w_{0}^{D}, q_{\varepsilon}^{D}-q_{0}^{D}\right) \mathbf{n} w_{0}^{D} d s .
\end{aligned}
$$

\section{Asymptotic behavior of the perturbed solutions}

In this section, we discuss the influence of the geometric perturbation $\mathcal{O}_{z, \varepsilon}$ on the solutions of the Stokes problems $\left(\mathcal{P}_{\varepsilon}^{N}\right)$ and $\left(\mathcal{P}_{\varepsilon}^{D}\right)$. More precisely, we derive an asymptotic formula describing the variations of the velocity and pressure fields with respect to the perturbation size $\varepsilon$. We start our analysis by the perturbed Stokes Neumann problem. 
KOHN-VOGELIUS FORMULATION AND HIGH-ORDER TOPOLOGICAL ASYMPTOTIC FORMULA FOR IDENTIFYING SMALL OBSTACLES IN A FLUID MEDIUM

\subsection{The Neumann perturbed solution}

This section is devoted to an asymptotic formula describing the variation of the Neumann solution $\left(w_{\varepsilon}^{N}-w_{0}^{N}\right)$ with respect $\varepsilon$. We begin our analysis by the following first order estimate.

Lemma 4.1. Let $\mathcal{O}_{z, \varepsilon}$ be a small geometric perturbation strictly included into $\Omega$. Then, the perturbed Stokes solution $\left(w_{\varepsilon}^{N}, q_{\varepsilon}^{N}\right)$ satisfies the behavior

$$
\begin{aligned}
w_{\varepsilon}^{N}(x)-w_{0}^{N}(x) & =E_{0}^{N}((x-z) / \varepsilon)+O(\varepsilon) \text { in } \Omega \backslash \overline{\mathcal{O}}_{z, \varepsilon}, \\
q_{\varepsilon}^{N}(x)-q_{0}^{N}(x) & =\frac{1}{\varepsilon} \Pi_{0}^{N}((x-z) / \varepsilon)+O(\varepsilon) \text { in } \Omega \backslash \overline{\mathcal{O}}_{z, \varepsilon}
\end{aligned}
$$

where the leading term $\left(E_{0}^{N}, \Pi_{0}^{N}\right)$ is solution to the following Stokes exterior problem

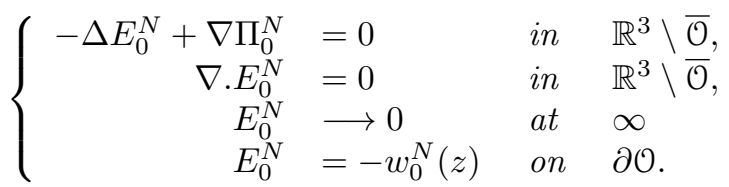

Proof: The existence of $\left(E_{0}^{N}, \Pi_{0}^{N}\right)$ can be established with the help of a single layer potential [33]. One can derive

$$
\begin{aligned}
& E_{0}^{N}(y)=\int_{\partial \mathcal{O}} U(y-t) \eta_{0}^{N}(t) d s(t), \forall y \in \mathbb{R}^{3} \backslash \overline{\mathcal{O}} \\
& \Pi_{0}^{N}(y)=\int_{\partial \mathcal{O}} P(y-t) \cdot \eta_{0}^{N}(t) d s(t), \forall y \in \mathbb{R}^{3} \backslash \overline{\mathcal{O}}
\end{aligned}
$$

where $(U, P)$ is the fundamental solution of the Stokes system in $\mathbb{R}^{3}$

$$
U(y)=\frac{1}{8 \pi \nu r}\left(I+e_{r} e_{r}^{T}\right), P(y)=\frac{y}{4 \pi r^{3}}, \quad \forall y \in \mathbb{R}^{3},
$$

with $r=\|y\|, e_{r}=\frac{y}{r}$ and $e_{r}^{T}$ is the transposed vector of $e_{r}$.

Here $\eta_{0}^{N}$ is the solution to the following boundary integral equation

$$
\int_{\partial \mathcal{O}} U(y-t) \eta_{0}^{N}(t) d s(t)=-w_{0}^{N}(z),, \forall y \in \partial \mathcal{O}
$$

Setting

$$
\begin{aligned}
& z_{\varepsilon}^{N}(x)=w_{\varepsilon}^{N}(x)-w_{0}^{N}(x)-E_{0}^{N}((x-z) / \varepsilon), \\
& s_{\varepsilon}^{N}(x)=q_{\varepsilon}^{N}(x)-q_{0}^{N}(x)-\frac{1}{\varepsilon} \Pi_{0}^{N}((x-z) / \varepsilon) .
\end{aligned}
$$


KOHN-VOGELIUS FORMULATION AND HIGH-ORDER TOPOLOGICAL ASYMPTOTIC FORMULA FOR IDENTIFYING SMALL OBSTACLES IN A FLUID MEDIUM

As one can observe, $\left(z_{\varepsilon}^{N}, s_{\varepsilon}^{N}\right)$ satisfies the system

$$
\left\{\begin{aligned}
-\nu \Delta z_{\varepsilon}^{N}+\nabla s_{\varepsilon}^{N} & =0 & & \text { in } \quad \Omega \backslash \overline{\mathcal{O}}_{z, \varepsilon} \\
\operatorname{div} z_{\varepsilon}^{N} & =0 & & \text { in } \quad \Omega \backslash \overline{\mathcal{O}}_{z, \varepsilon}, \\
\sigma\left(z_{\varepsilon}^{N}, s_{\varepsilon}^{N}\right) \mathbf{n} & =-\frac{1}{\varepsilon} \sigma\left(E_{0}^{N}, \Pi_{0}^{N}\right)((x-z) / \varepsilon) \mathbf{n} & & \text { on } \quad \Sigma, \\
w_{\varepsilon}^{N} & =-E_{0}^{N}((x-z) / \varepsilon) & & \text { on } \quad \Gamma, \\
w_{\varepsilon}^{N} & =-w_{0}^{N}+w_{0}^{N}(z) & & \text { on } \partial \mathcal{O}_{z, \varepsilon} .
\end{aligned}\right.
$$

Using the change of variable $x=z+\varepsilon y$ and the standard energy estimate for the Stokes problem, one can derive that there exists a constant $c>0$, independent of $\varepsilon$, such that

$$
\left\|z_{\varepsilon}^{N}\right\|_{H^{1}\left(\Omega \backslash \overline{\mathcal{O}}_{z, \varepsilon}\right)}+\left\|s_{\varepsilon}^{N}\right\|_{L^{2}\left(\Omega \backslash \overline{\mathcal{O}}_{z, \varepsilon}\right)} \leq c \varepsilon .
$$

One can see ([19], Proposition 3.1) for similar proof.

Next, we extend this estimate to the high-order case. The obtained asymptotic behavior is illustrated by the following theorem.

Theorem 4.2. Let $\mathcal{O}_{z, \varepsilon}=z+\varepsilon \omega$ be a small geometric perturbation, strictly embedded in the fluid flow domain $\Omega$. Then, the velocity and pressure fields satisfy the following asymptotic behavior

$$
\begin{gathered}
\left.w_{\varepsilon}^{N}(x)-w_{0}^{N}(x)=E_{0}^{N}((x-z) / \varepsilon)+\sum_{k=1}^{m} \varepsilon^{k}\left[V_{k}^{N}(x)+E_{k}^{N}((x-z) / \varepsilon)\right)\right]+o\left(\varepsilon^{m}\right), \quad(7) \\
\left.q_{\varepsilon}^{N}(x)-q_{0}^{N}(x)=\frac{1}{\varepsilon} \Pi_{0}^{N}((x-z) / \varepsilon)+\sum_{k=1}^{m} \varepsilon^{k}\left[S_{k}^{N}(x)+\frac{1}{\varepsilon} \Pi_{k}^{N}((x-z) / \varepsilon)\right)\right]+o\left(\varepsilon^{m}\right),
\end{gathered}
$$

where $\left(V_{k}^{N}, S_{k}^{N}\right)_{0 \leq k \leq m}$ is a set of smooth functions satisfying the Stokes system in $\Omega$ and $\left(E_{k}^{N}, \Pi_{k}^{N}\right)_{0 \leq k \leq m}$ is a set of smooth functions verifying the exterior Stokes problem in $\mathbb{R}^{3} \backslash \overline{\mathcal{O}}$.

Proof: The terms of the derived asymptotic expansion are built iteratively.

Initialization: We start our construction process by the terms associated with $k=0$. The two sequences $\left(V_{k}^{N}, S_{k}^{N}\right)_{0 \leq k \leq m}$ and $\left(E_{k}^{N}, \Pi_{k}^{N}\right)_{0 \leq k \leq m}$ are initialized as follows: 
KOHN-VOGELIUS FORMULATION AND HIGH-ORDER TOPOLOGICAL ASYMPTOTIC FORMULA FOR IDENTIFYING SMALL OBSTACLES IN A FLUID MEDIUM

$-\left(V_{0}^{N}, S_{0}^{N}\right)=\left(w_{0}^{N}, q_{0}^{N}\right)$ which is the solution to the Stokes problem $\left(\mathcal{P}_{0}^{N}\right)$, defined in

the non perturbed domain $\Omega$. $\mathbb{R}^{3} \backslash \overline{\mathcal{O}}$

$-\left(E_{0}^{N}, \Pi_{0}^{N}\right)$ is the solution to the exterior Stokes problem (6), defined in

The $k^{t h}$ term : Let $k \in\{1, \ldots, m\}$. Assume that we have already derived the terms $\left(V_{i}^{N}, S_{i}^{N}\right)$ and $\left(E_{i}^{N}, \Pi_{i}^{N}\right)$ for all $0 \leq i \leq k-1$, and we want to derive the terms $\left(V_{k}^{N}, S_{k}^{N}\right)$ and $\left(E_{k}^{N}, \Pi_{k}^{N}\right)$.

In order to define the desired terms, we need to establish a preliminary calculus. It concerns the asymptotic behavior of the functions $E_{i}^{N}$ and $\Pi_{i}^{N}$ with respect to $\varepsilon$.

Recalling that $\left(E_{i}^{N}, \Pi_{i}^{N}\right)$ is constructed as a solution to an exterior Stokes problem defined in $\mathbb{R}^{3} \backslash \overline{\mathcal{O}}$. Then, due to a single layer potential [33], $\left(E_{i}^{N}, \Pi_{i}^{N}\right)$ can be written as

$$
\begin{aligned}
& E_{i}^{N}(y)=\int_{\partial \mathcal{O}} U(y-t) \eta_{i}^{N}(t) d s(t), \forall y \in \mathbb{R}^{3} \backslash \overline{\mathcal{O}} \\
& \Pi_{i}^{N}(y)=\int_{\partial \mathcal{O}} P(y-t) \cdot \eta_{i}^{N}(t) d s(t), \forall y \in \mathbb{R}^{3} \backslash \overline{\mathcal{O}}
\end{aligned}
$$

where $\eta_{i}^{N}$ is the solution to a boundary integral equation defined on $\partial \mathcal{O}$.

From the fact that $U(y / \varepsilon)=\varepsilon U(y)$ it follows that for each $x \in \mathbb{R}^{3} \backslash \overline{\mathcal{O}_{z, \varepsilon}}$ we have

$$
\begin{aligned}
E_{i}^{N}((x-z) / \varepsilon) & =\int_{\partial \mathcal{O}} U((x-z) / \varepsilon-t) \eta_{i}^{N}(t) d s(t) \\
& =\varepsilon \int_{\partial \mathcal{O}} U((x-z)-\varepsilon t) \eta_{i}^{N}(t) d s(t) .
\end{aligned}
$$

From the fact that $\mathcal{O}_{z, \varepsilon}$ is not close to the boundary $\partial \Omega$, one can remark that for all $t \in \partial \mathcal{O}$, the function $\varepsilon \longmapsto U_{x-z, t}(\varepsilon)=\varepsilon U((x-z)-\varepsilon t)$ is smooth with respect to $\varepsilon$ and admits the following asymptotic expansion

$$
U_{x-z, t}(\varepsilon)=\sum_{p=1}^{m} \frac{\varepsilon^{p}}{(p-1) !} U_{x-z, t}^{(p-1)}(0)+o\left(\varepsilon^{m}\right) \text {, }
$$

where $U_{x-z, t}^{(p)}(0)$ is the $p^{\text {th }}$ derivative of $U_{x-z, t}$ at $\varepsilon=0$. It depends on the $p^{\text {th }}$ derivative of the function $U$ at the point $x-z$. Consequently, the function 
KOHN-VOGELIUS FORMULATION AND HIGH-ORDER TOPOLOGICAL ASYMPTOTIC FORMULA FOR IDENTIFYING SMALL OBSTACLES IN A

$\varepsilon \longmapsto E_{i}^{N}((x-z) / \varepsilon)$ satisfies the following asymptotic behavior

$$
E_{i}^{N}((x-z) / \varepsilon)=\sum_{p=1}^{m} \varepsilon^{p} E_{N, i}^{(p)}(x-z)+o\left(\varepsilon^{m}\right),
$$

with $E_{N, i}^{(p)}$ is the smooth function defined in $\mathbb{R}^{3} \backslash \overline{\mathcal{O}}$ by

$$
E_{N, i}^{(p)}(y)=\frac{1}{(p-1) !} \int_{\partial \mathcal{O}} U_{y, t}^{(p-1)}(0) \eta_{i}^{N}(t) d s(t), \forall y \in \mathbb{R}^{3} \backslash \overline{\mathcal{O}} .
$$

Similarly, we applied the same analysis for the pressure field. Then, using the fact that $P(y / \varepsilon)=\varepsilon^{2} P(y)$ one can obtain

$$
\Pi_{i}^{N}((x-z) / \varepsilon)=\varepsilon^{2} \int_{\partial \mathcal{O}} P((x-z)-\varepsilon t) . \eta_{i}^{N}(t) d s(t) .
$$

From the smoothness of the function $\varepsilon \longmapsto P_{x-z, t}(\varepsilon)=\varepsilon^{2} P((x-z)-\varepsilon t)$ with respect to $\varepsilon$, it follows

$$
P_{x-z, t}(\varepsilon)=\sum_{q=1}^{m-1} \frac{\varepsilon^{q+1}}{(q-1) !} P_{x-z, t}^{(q-1)}(0)+o\left(\varepsilon^{m}\right),
$$

where $P_{x-z, t}^{(q)}(0)$ is the $q^{\text {th }}$ derivative of $P_{x-z, t}$ at $\varepsilon=0$. Hence, we have

$$
\Pi_{i}^{N}((x-z) / \varepsilon)=\sum_{q=1}^{m-1} \varepsilon^{q+1} \Pi_{N, i}^{(q)}(x-z)+o\left(\varepsilon^{m}\right),
$$

with $\Pi_{N, i}^{(q)}$ is the smooth function defined in $\mathbb{R}^{3} \backslash \overline{\mathcal{O}}$ by

$$
\Pi_{N, i}^{(q)}(y)=\frac{1}{(q-1) !} \int_{\partial \mathcal{O}} P_{y, t}^{(q-1)}(0) . \eta_{i}^{N}(t) d s(t), \forall y \in \mathbb{R}^{3} \backslash \overline{\mathcal{O}}
$$

-Determining the term $\left(V_{k}^{N}, S_{k}^{N}\right)$. It is constructed using the functions $E_{i}^{N}, 0 \leq i \leq k-1$. It is defined as the solution to the following Stokes system

$$
\left\{\begin{array}{rlrl}
-\Delta V_{k}^{N}+\nabla S_{k}^{N} & =0 & \text { in } & \Omega, \\
\nabla \cdot V_{k}^{N} & =0 & \text { in } & \Omega, \\
\sigma\left(V_{k}^{N}, S_{k}^{N}\right) \mathbf{n} & =-\sum_{p=1}^{k} \sigma\left(E_{N, k-p}^{(p)}, \Pi_{N, k-p}^{(p)}\right)(x-z) \mathbf{n} & \text { on } \quad \Sigma, \\
V_{k}^{N} & =-\sum_{p=1}^{k} E_{N, k-p}^{(p)}(x-z) & \text { on } \quad \Gamma,
\end{array}\right.
$$


KOHN-VOGELIUS FORMULATION AND HIGH-ORDER TOPOLOGICAL ASYMPTOTIC FORMULA FOR IDENTIFYING SMALL OBSTACLES IN A FLUID MEDIUM

where the functions $E_{N, j}^{(p)}$ and $\Pi_{N, j}^{(p)}$ are defined by (10) and (12).

-Determining the term $\left(E_{k}^{N}, \Pi_{k}^{N}\right)$. It is constructed using the functions $V_{i}^{N}, 0 \leq i \leq k$. This term is defined as a solution to the following exterior problem

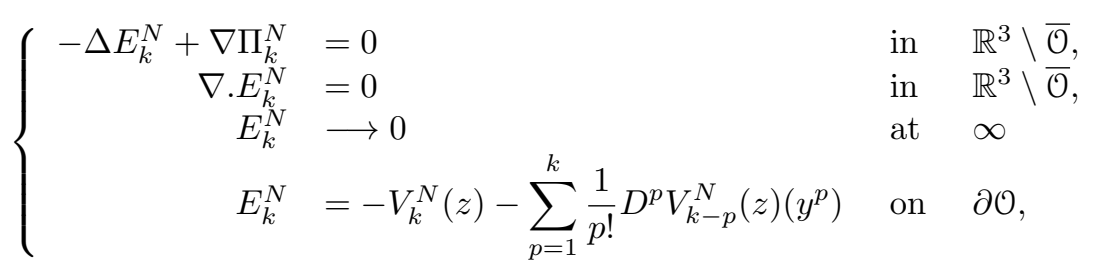

where $D^{p} V_{k-p}^{N}(z)$ is the $p^{\text {th }}$ derivative of the function $V_{k-p}^{N}$ at the point $z$ and $y^{p}=(y, \ldots, y) \in\left(\mathbb{R}^{3}\right)^{p}$.

Justification of the asymptotic formulas: Here we will prove that the constructed sequences $\left(V_{k}^{N}, S_{k}^{N}\right)_{0 \leq k \leq m}$ and $\left(E_{k}^{N}, \Pi_{k}^{N}\right)_{0 \leq k \leq m}$ permit us to derive the expected asymptotic formulas.

Posing

$$
\begin{aligned}
& \left.\left.R_{m, \varepsilon}^{N}(x)=w_{0}^{N}(x)+E_{0}^{N}((x-z) / \varepsilon)\right)+\sum_{k=1}^{m} \varepsilon^{k}\left[V_{k}^{N}(x)+E_{k}^{N}((x-z) / \varepsilon)\right)\right]-w_{\varepsilon}^{N}, \\
& \left.\left.\xi_{m, \varepsilon}^{N}(x)=S_{0}^{N}(x)+\frac{1}{\varepsilon} \Pi_{0}^{N}((x-z) / \varepsilon)\right)+\sum_{k=1}^{m} \varepsilon^{k}\left[S_{k}^{N}(x)+\frac{1}{\varepsilon} \Pi_{k}^{N}((x-z) / \varepsilon)\right)\right]-q_{\varepsilon}^{N} .
\end{aligned}
$$

One can easily verify that $\left(R_{m, \varepsilon}^{N}, \xi_{m, \varepsilon}^{N}\right)$ solves the Stokes system in $\Omega \backslash \overline{\mathcal{O}}_{z, \varepsilon}$

$$
\begin{aligned}
& -\Delta R_{m, \varepsilon}^{N}+\nabla \xi_{m, \varepsilon}^{N}=0 \quad \text { in } \Omega \backslash \overline{\mathcal{O}}_{z, \varepsilon}, \\
& \nabla \cdot R_{m, \varepsilon}^{N}=0 \quad \text { in } \Omega \backslash \overline{\mathcal{O}}_{z, \varepsilon},
\end{aligned}
$$

and satisfies the following boundaries conditions:

- On $\partial \mathcal{O}_{z, \varepsilon}$ : Using the systems (13)-(14), the multi-linearity of $D^{p} V_{k-p}^{N}(z)$, Taylor's Theorem and the fact that $\|x-z\|=O(\varepsilon)$ on $\partial \mathcal{O}_{z, \varepsilon}$, one can derive

$$
R_{m, \varepsilon}^{N}(x)=\sum_{k=0}^{m} \varepsilon^{k}\left[V_{k}^{N}(x)-\sum_{p=0}^{m-k} \frac{1}{p !} D^{p} V_{k}^{N}(z)\left((x-z)^{p}\right)\right]=o\left(\varepsilon^{m}\right) .
$$


- On $\Gamma$ : the Dirichlet boundary condition in (13) and the asymptotic expansions (9) and (11) imply

$$
\begin{aligned}
R_{m, \varepsilon}^{N}(x) & \left.=\sum_{k=0}^{m} \varepsilon^{k} E_{k}^{N}((x-z) / \varepsilon)\right)+\sum_{k=1}^{m} \varepsilon^{k} V_{k}^{N}(x) \\
& =\sum_{k=0}^{m} \varepsilon^{k}\left[\sum_{p=1}^{m} \varepsilon^{p} E_{N, k}^{(p)}(x-z)\right]-\sum_{k=1}^{m} \varepsilon^{k}\left[\sum_{p=1}^{k} E_{N, k-p}^{(p)}(x-z)\right]+o\left(\varepsilon^{m}\right) \\
& =o\left(\varepsilon^{m}\right) .
\end{aligned}
$$

- On $\Sigma$ : by the change of variable $x=z+\varepsilon y$, we have

$$
\sigma\left(R_{m, \varepsilon}, \xi_{m, \varepsilon}\right)=\frac{1}{\varepsilon} \sum_{k=0}^{m} \varepsilon^{k} \sigma_{y}\left(E_{k}^{N}, \Pi_{k}^{N}\right)((x-z) / \varepsilon)+\sum_{k=1}^{m} \varepsilon^{k} \sigma\left(V_{k}^{N}, S_{k}^{N}\right)(x)
$$

Using again the change of variable, from (9) and (11) one can deduce

$$
\sigma_{y}\left(E_{k}^{N}, \Pi_{k}^{N}\right)((x-z) / \varepsilon)=\varepsilon \sum_{p=1}^{m} \varepsilon^{p} \sigma\left(E_{N, k}^{(p)}, \Pi_{N, k}^{(p)}\right)(x-z)+o\left(\varepsilon^{m}\right) .
$$

The two last relations combined with the Neumann condition used in (13) imply

$$
\sigma\left(R_{m, \varepsilon}, \xi_{m, \varepsilon}\right) \mathbf{n}=o\left(\varepsilon^{m}\right) \text { on } \Sigma \text {. }
$$

\subsection{The Dirichlet perturbed solution}

This section is concerned with brief analysis of the Dirichlet case. Here, we consider $\left(V_{k}^{D}, S_{k}^{D}\right)_{0 \leq k \leq m}$ a set of smooth functions satisfying the Stokes system in $\Omega$ and $\left(E_{k}^{D}, \Pi_{k}^{D}\right)_{0 \leq k \leq m}$ a set of smooth functions verifying the exterior Stokes problem in $\mathbb{R}^{3} \backslash \overline{\mathcal{O}}$. The two considered sequences $\left(V_{k}^{D}, S_{k}^{D}\right)_{0 \leq k \leq m}$ and $\left(E_{k}^{D}, \Pi_{k}^{D}\right)_{0 \leq k \leq m}$ are initialized as follows:

$-\left(V_{0}^{D}, S_{0}^{\bar{D}}\right)=\left(w_{0}^{D}, q_{0}^{D}\right)$ which is the solution to the Stokes problem $\left(\mathcal{P}_{0}^{D}\right)$.

- $\left(E_{0}^{D}, \Pi_{0}^{D}\right)$ is defined as the solution to the following exterior Stokes problem

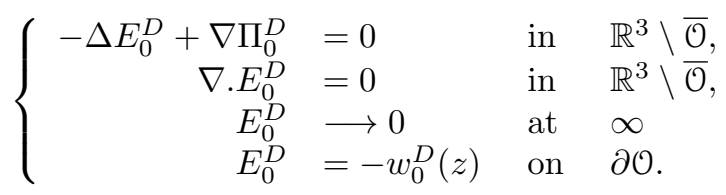


KOHN-VOGELIUS FORMULATION AND HIGH-ORDER TOPOLOGICAL ASYMPTOTIC FORMULA FOR IDENTIFYING SMALL OBSTACLES IN A

- The term $\left(V_{k}^{D}, S_{k}^{D}\right)$. It is defined as the unique solution to the following Stokes system

$$
\left\{\begin{aligned}
-\Delta V_{k}^{D}+\nabla S_{k}^{D} & =0 & & \text { in } \quad \Omega, \\
\nabla \cdot V_{k}^{D} & =0 & & \text { in } \quad \Omega, \\
V_{k}^{D} & =-\sum_{p=1}^{k} E_{D, k-p}^{(p)}(x-z) & & \text { on } \quad \Gamma \cup \Sigma,
\end{aligned}\right.
$$

where the functions $E_{D, j}^{(p)}, 0 \leq j \leq k$ are defined by

$$
E_{D, j}^{(p)}(y)=\frac{1}{(p-1) !} \int_{\partial \mathcal{O}} U_{y, t}^{(p-1)}(0) \eta_{j}^{D}(t) d s(t), \forall y \in \mathbb{R}^{3} \backslash \overline{\mathcal{O}} .
$$

- The term $\left(E_{k}^{D}, \Pi_{k}^{D}\right)$. It is constructed using the functions $V_{j}^{D}, 0 \leq j \leq k$. This term is defined as a solution to the following exterior problem

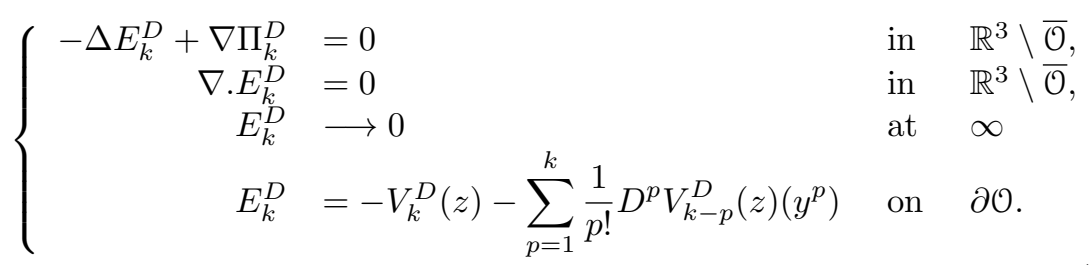

Theorem 4.3. In the presence of a small geometric perturbation $\mathcal{O}_{z, \varepsilon}=z+\varepsilon \omega$ inside the fluid flow domain $\Omega$, the solution $\left(w_{\varepsilon}^{D}, q_{\varepsilon}^{D}\right)$ of the perturbed Dirichlet Stokes problem admits the asymptotic behavior

$\left.w_{\varepsilon}^{D}(x)-w_{0}^{D}(x)=E_{0}^{D}((x-z) / \varepsilon)+\sum_{k=1}^{m} \varepsilon^{k}\left[V_{k}^{D}(x)+E_{k}^{D}((x-z) / \varepsilon)\right)\right]+o\left(\varepsilon^{m}\right)$,

$\left.q_{\varepsilon}^{D}(x)-q_{0}^{D}(x)=\frac{1}{\varepsilon} \Pi_{0}^{D}((x-z) / \varepsilon)+\sum_{k=1}^{m} \varepsilon^{k}\left[S_{k}^{D}(x)+\frac{1}{\varepsilon} \Pi_{k}^{D}((x-z) / \varepsilon)\right)\right]+o\left(\varepsilon^{m}\right)$.

\section{High-order topological asymptotic expansion}

In this section we extend the topological derivative notion $[16,17,18,19,20$, $21,34]$ for the high-order case. We derive a high-order terms in the topological asymptotic expansion for the Stokes operator. We will derive an asymptotic 
KOHN-VOGELIUS FORMULATION AND HIGH-ORDER TOPOLOGICAL ASYMPTOTIC FORMULA FOR IDENTIFYING SMALL OBSTACLES IN A

formula describing the variation of the Kohn-Vogelius functional $\mathcal{K}$ with respect to the insertion of a small obstacle inside the fluid flow domain $\Omega$.

From Theorem 3.1, the variation caused by the presence of the geometric perturbation $\mathcal{O}_{z, \varepsilon}=z+\varepsilon \mathcal{O}$ can be decomposed as

$$
\mathcal{K}\left(\Omega \backslash \overline{\mathcal{O}}_{z, \varepsilon}\right)-\mathcal{K}(\Omega)=\mathcal{J}^{N}(\varepsilon)-\mathcal{J}^{D}(\varepsilon),
$$

where the Neumann and Dirichlet terms are defined by

$$
\begin{aligned}
& \mathcal{J}^{N}(\varepsilon)=\int_{\partial \mathcal{O}_{z, \varepsilon}} \sigma\left(w_{\varepsilon}^{N}-w_{0}^{N}, q_{\varepsilon}^{N}-q_{0}^{N}\right) \mathbf{n} w_{0}^{N} d s-\nu \int_{\mathcal{O}_{z, \varepsilon}}\left|\nabla w_{0}^{N}\right|^{2} d x,(22) \\
& \partial^{D}(\varepsilon)=\int_{\partial \mathcal{O}_{z, \varepsilon}} \sigma\left(w_{\varepsilon}^{D}-w_{0}^{D}, q_{\varepsilon}^{D}-q_{0}^{D}\right) \mathbf{n} w_{0}^{D} d s-\nu \int_{\mathcal{O}_{z, \varepsilon}}\left|\nabla w_{0}^{D}\right|^{2} d x .
\end{aligned}
$$

To derive the expected high-order asymptotic expansion for Kohn-Vogelius functional $\mathcal{K}$ we will examine the terms $\mathcal{J}^{N}$ and $\mathcal{J}^{D}$ separately.

\subsection{Estimate of the Neumann terms}

Here, we derive a sensitivity analysis for each term in $g^{N}(\varepsilon)$ with respect to the parameter $\varepsilon$. We will establish a high-order asymptotic expansion for each term. Our mathematical analysis is based on the asymptotic behavior of the perturbed solution $\left(w_{\varepsilon}^{N}, q_{\varepsilon}^{N}\right)$.

Lemma 5.1. The first term in (22) admits the estimate

$$
\begin{aligned}
\int_{\partial \mathcal{O}_{z, \varepsilon}} & \sigma\left(w_{\varepsilon}^{N}-w_{0}^{N}, q_{\varepsilon}^{N}-q_{0}^{N}\right) \mathbf{n} \cdot w_{0}^{N} d s \\
& =\sum_{k=0}^{m-1} \varepsilon^{k+1} \int_{\partial \mathcal{O}} \sigma_{y}\left(E_{k}^{N}, \Pi_{k}^{N}\right)(y) \mathbf{n}(y) \cdot w_{0}^{N}(z+\varepsilon y) d s(y) \\
& +\sum_{k=1}^{m-2} \varepsilon^{k+2} \int_{\partial \mathcal{O}} \sigma\left(V_{k}^{N}, S_{k}^{N}\right)(z+\varepsilon y) \mathbf{n} \cdot w_{0}^{N}(z+\varepsilon y) d s(y)+o\left(\varepsilon^{m}\right) .
\end{aligned}
$$

Proof: From the change of variable $x=z+\varepsilon y$, it follows

$$
\left.\left.\nabla_{x}\left(E_{k}^{N}((x-z) / \varepsilon)\right)\right)=\frac{1}{\varepsilon} \nabla_{y} E_{k}^{N}((x-z) / \varepsilon)\right), \quad 0 \leq k \leq m,
$$

where $\nabla_{\xi}$ denotes the gradient with respect to the variable $\xi(\xi=x$ or $y)$. 
KOHN-VOGELIUS FORMULATION AND HIGH-ORDER TOPOLOGICAL ASYMPTOTIC FORMULA FOR IDENTIFYING SMALL OBSTACLES IN A FLUID MEDIUM

Using the previous relation and the decomposition presented in Theorem 4.2, one can derive

$$
\begin{aligned}
\sigma\left(w_{\varepsilon}^{N}-w_{0}^{N}, q_{\varepsilon}^{N}-q_{0}^{N}\right)= & \left.\frac{1}{\varepsilon} \sigma_{y}\left(E_{0}^{N}, \Pi_{0}^{N}\right)((x-z) / \varepsilon)\right)+\sum_{k=1}^{m} \varepsilon^{k} \sigma\left(V_{k}^{N}, S_{k}^{N}\right)(x) \\
& +\sum_{k=1}^{m+1} \varepsilon^{k-1} \sigma_{y}\left(E_{k}^{N}, \Pi_{k}^{N}\right)((x-z) / \varepsilon)+o\left(\varepsilon^{m}\right) .
\end{aligned}
$$

Then, the first term in (22) satisfies the estimate

$$
\begin{aligned}
\int_{\partial \mathcal{O}_{z, \varepsilon}} \sigma\left(w_{\varepsilon}^{N}-w_{0}^{N}, q_{\varepsilon}^{N}-q_{0}^{N}\right) \mathbf{n} . w_{0}^{N} d s & \left.=\frac{1}{\varepsilon} \int_{\partial \mathcal{O}_{z, \varepsilon}} \sigma_{y}\left(E_{0}^{N}, \Pi_{0}^{N}\right)((x-z) / \varepsilon)\right) \mathbf{n} \cdot w_{0}^{N} d s \\
& +\sum_{k=1}^{m+1} \varepsilon^{k-1} \int_{\partial \mathcal{O}_{z, \varepsilon}} \sigma_{y}\left(E_{k}^{N}, \Pi_{k}^{N}\right)((x-z) / \varepsilon) \mathbf{n} \cdot w_{0}^{N} d s \\
& +\sum_{k=1}^{m} \varepsilon^{k} \int_{\partial \mathcal{O}_{z, \varepsilon}} \sigma\left(V_{k}^{N}, S_{k}^{N}\right)(x) \mathbf{n} \cdot w_{0}^{N} d s+o\left(\varepsilon^{m}\right) .
\end{aligned}
$$

Making use of the change of variable $x=z+\varepsilon y$, one can deduce

$$
\begin{aligned}
& \int_{\partial \mathcal{O}_{z, \varepsilon}} \sigma_{y}\left(E_{k}^{N}, \Pi_{k}^{N}\right)((x-z) / \varepsilon) \mathbf{n} \cdot w_{0}^{N} d s=\varepsilon^{2} \int_{\partial \mathcal{O}} \sigma_{y}\left(E_{k}^{N}, \Pi_{k}^{N}\right)(y) \mathbf{n}(y) \cdot w_{0}^{N}(z+\varepsilon y) d s(y), \\
& \int_{\partial \mathcal{O}_{z, \varepsilon}} \sigma\left(V_{k}^{N}, S_{k}^{N}\right)(x) \mathbf{n} \cdot w_{0}^{N} d s=\varepsilon^{2} \int_{\partial \mathcal{O}} \sigma\left(V_{k}^{N}, S_{k}^{N}\right)(z+\varepsilon y) \mathbf{n}(y) \cdot w_{0}^{N}(z+\varepsilon y) d s(y) .
\end{aligned}
$$

Consequently, we obtain

$$
\begin{aligned}
& \int_{\partial \mathcal{O}_{z, \varepsilon}} \sigma\left(w_{\varepsilon}^{N}-w_{0}^{N}, q_{\varepsilon}^{N}-q_{0}^{N}\right) \mathbf{n} \cdot w_{0}^{N} d s=\sum_{k=0}^{m-1} \varepsilon^{k+1} \int_{\partial \mathcal{O}} \sigma_{y}\left(E_{k}^{N}, \Pi_{k}^{N}\right)(y) \mathbf{n}(y) \cdot w_{0}^{N}(z+\varepsilon y) d s(y) \\
& +\sum_{k=1}^{m-2} \varepsilon^{k+2} \int_{\partial \mathcal{O}} \sigma\left(V_{k}^{N}, S_{k}^{N}\right)(z+\varepsilon y) \mathbf{n} \cdot w_{0}^{N}(z+\varepsilon y) d s(y)+o\left(\varepsilon^{m}\right) .
\end{aligned}
$$

Next, we will examine the two integral terms in the right hand side of (24).

Lemma 5.2. We have

$$
\sum_{k=0}^{m-1} \varepsilon^{k+1} \int_{\partial \mathcal{O}} \sigma_{y}\left(E_{k}^{N}, \Pi_{k}^{N}\right)(y) \mathbf{n}(y) \cdot w_{0}^{N}(z+\varepsilon y) d s(y)=\sum_{q=0}^{m-1} \varepsilon^{q+1} \mathcal{K}_{q}^{1, N}(z, \mathcal{O})+o\left(\varepsilon^{m}\right),
$$


KOHN-VOGELIUS FORMULATION AND HIGH-ORDER TOPOLOGICAL ASYMPTOTIC FORMULA FOR IDENTIFYING SMALL OBSTACLES IN A FLUID MEDIUM

where the functions $z \longmapsto \mathcal{K}_{q}^{1, N}(z, \mathcal{O}), 0 \leq q \leq m$ are defined in $\Omega$ by

$$
\mathcal{K}_{q}^{1, N}(z, \mathcal{O})=\sum_{p=0}^{q} \frac{1}{p !} \int_{\partial \mathcal{O}} \sigma_{y}\left(E_{q-p}^{N}, \Pi_{q-p}^{N}\right)(y) \mathbf{n}(y) \cdot\left[D^{(p)} w_{0}^{N}(z)\left(y^{p}\right)\right] d s(y)
$$

with $D^{(p)} w_{0}^{N}(z)$ denotes the $p^{\text {th }}$ derivative of the function $w_{0}^{N}$ at the point $z \in \Omega$ and $y^{p}=(y, \ldots, y) \in\left(\mathbb{R}^{3}\right)^{p}$.

Proof: Due to the smoothness of the velocity field $w_{0}^{N}$, by Taylor's theorem one can derive

$$
w_{0}^{N}(z+\varepsilon y)=\sum_{p=0}^{m} \frac{\varepsilon^{p}}{p !} D^{(p)} w_{0}^{N}(z)\left(y^{p}\right)+o\left(\varepsilon^{m}\right) .
$$

It follows

$$
\begin{aligned}
& \sum_{k=0}^{m-1} \varepsilon^{k+1} \int_{\partial \mathcal{O}} \sigma_{y}\left(E_{k}^{N}, \Pi_{k}^{N}\right)(y) \mathbf{n}(y) \cdot w_{0}^{N}(z+\varepsilon y) d s(y) \\
= & \sum_{k=0}^{m-1} \varepsilon^{k+1}\left(\sum_{p=0}^{m} \frac{\varepsilon^{p}}{p !} \int_{\partial \mathcal{O}} \sigma_{y}\left(E_{k}^{N}, \Pi_{k}^{N}\right)(y) \mathbf{n}(y) \cdot\left[D^{(p)} w_{0}^{N}(z)\left(y^{p}\right)\right] d s(y)\right)+o\left(\varepsilon^{m}\right) \\
= & \sum_{q=0}^{m-1} \varepsilon^{q+1}\left(\sum_{p=0}^{q} \frac{1}{p !} \int_{\partial \mathcal{O}} \sigma_{y}\left(E_{q-p}^{N}, \Pi_{q-p}^{N}\right)(y) \mathbf{n}(y) \cdot\left[D^{(p)} w_{0}^{N}(z)\left(y^{p}\right)\right] d s(y)\right)+o\left(\varepsilon^{m}\right)
\end{aligned}
$$

Lemma 5.3. The second integral term in the right hand side of (24) satisfies

$$
\sum_{k=1}^{m-2} \varepsilon^{k+2} \int_{\partial \mathcal{O}} \sigma\left(V_{k}^{N}, S_{k}^{N}\right)(z+\varepsilon y) \mathbf{n} \cdot w_{0}^{N}(z+\varepsilon y) d s(y)=\sum_{q=1}^{m-2} \varepsilon^{q+2} \mathcal{K}_{q}^{2, N}(z, \mathcal{O})+o\left(\varepsilon^{m}\right) .
$$

where the functions $z \longmapsto \mathcal{K}_{q}^{2, N}(z, \mathcal{O}), 1 \leq q \leq m-2$ are defined in $\Omega$ by

$$
\mathcal{K}_{q}^{2, N}(z, \mathcal{O})=\sum_{p=0}^{q-1} \sum_{l=0}^{p} \frac{1}{l !(p-l) !} \int_{\partial \mathcal{O}} \sigma^{(l)}\left(V_{q-p}^{N}, S_{q-p}^{N}\right)(z) \mathbf{n} \cdot D^{(p-l)} w_{0}^{N}(z)\left(y^{p-l}\right) d s(y)
$$

Proof: Here we exploit the smoothness of the functions $V_{q}^{N}$ and $S_{q}^{N}$. This follows from the fact that $\left(V_{q}^{N}, S_{q}^{N}\right)$ is solution to the Stokes problem (13), 
KOHN-VOGELIUS FORMULATION AND HIGH-ORDER TOPOLOGICAL ASYMPTOTIC FORMULA FOR IDENTIFYING SMALL OBSTACLES IN A FLUID MEDIUM

defined in a smooth domain $\Omega$ and verifying smooth boundary data on $\partial \Omega$. Using Taylor's formula, one can derive

$$
\begin{aligned}
S_{k}^{N}(z+\varepsilon y) & =\sum_{p=0}^{m} \frac{\varepsilon^{p}}{p !} D^{(p)}\left(S_{k}^{N}\right)(z)\left(y^{p}\right)+o\left(\varepsilon^{m}\right), \\
\frac{\partial\left(V_{k}^{N}\right)_{i}}{\partial x_{j}}(z+\varepsilon y) & =\sum_{p=0}^{m} \frac{\varepsilon^{p}}{p !} D^{(p)}\left(\frac{\partial\left(V_{k}^{N}\right)_{i}}{\partial x_{j}}\right)(z)\left(y^{p}\right)+o\left(\varepsilon^{m}\right), 1 \leq i, j \leq 3 .
\end{aligned}
$$

Recalling that

$$
\sigma\left(V_{k}^{N}, S_{k}^{N}\right)_{i, j}=\mu\left(\frac{\partial\left(V_{k}^{N}\right)_{i}}{\partial x_{j}}+\frac{\partial\left(V_{k}^{N}\right)_{j}}{\partial x_{i}}\right)-S_{k}^{N} \delta_{i, j}
$$

where $\delta_{i, j}$ is the Kronecker symbol.

Then, for each $1 \leq i, j \leq 3$, one can derive

$$
\sigma\left(V_{k}^{N}, S_{k}^{N}\right)_{i, j}(z+\varepsilon y)=\sum_{p=0}^{m} \frac{\varepsilon^{p}}{p !} \sigma_{i, j}^{(p)}\left(V_{k}^{N}, S_{k}^{N}\right)(z)+o\left(\varepsilon^{m}\right),
$$

with $\sigma_{i, j}^{(p)}\left(V_{k}^{N}, S_{k}^{N}\right)(z)=D^{(p)}\left(\sigma\left(V_{k}^{N}, S_{k}^{N}\right)_{i, j}\right)(z)$ which is the $p^{\text {th }}$ derivative of the function $\mu\left(\frac{\partial\left(V_{k}^{N}\right)_{i}}{\partial x_{j}}+\frac{\partial\left(V_{k}^{N}\right)_{j}}{\partial x_{i}}\right)-S_{k}^{N} \delta_{i, j}$ at the point $z \in \Omega$.

Due to the Cauchy product formula, the relations (25) and (26) imply

$$
\begin{gathered}
\int_{\partial \mathcal{O}} \sigma\left(V_{k}^{N}, S_{k}^{N}\right)(z+\varepsilon y) \mathbf{n} \cdot w_{0}^{N}(z+\varepsilon y) d s(y)= \\
\sum_{p=0}^{m} \varepsilon^{p}\left(\sum_{l=0}^{p} \frac{1}{l !(p-l) !} \int_{\partial \mathcal{O}} \sigma^{(l)}\left(V_{k}^{N}, S_{k}^{N}\right)(z) \mathbf{n} \cdot D^{(p-l)} w_{0}^{N}(z)\left(y^{p-l}\right) d s(y)\right)+o\left(\varepsilon^{m}\right) .
\end{gathered}
$$

Finally, we obtain

$$
\begin{aligned}
& \sum_{k=1}^{m-2} \varepsilon^{k+2} \int_{\partial \mathcal{O}} \sigma\left(V_{k}^{N}, S_{k}^{N}\right)(z+\varepsilon y) \mathbf{n} \cdot w_{0}^{N}(z+\varepsilon y) d s(y)= \\
& \sum_{q=1}^{m-2} \varepsilon^{q+2}\left(\sum_{p=0}^{q-1} \sum_{l=0}^{p} \frac{1}{l !(p-l) !} \int_{\partial \mathcal{O}} \sigma^{(l)}\left(V_{q-p}^{N}, S_{q-p}^{N}\right)(z) \mathbf{n} \cdot D^{(p-l)} w_{0}^{N}(z)\left(y^{p-l}\right) d s(y)\right)+o\left(\varepsilon^{m}\right) .
\end{aligned}
$$

Lemma 5.4. The second term in (22) admits the estimate

$$
\nu \int_{\mathcal{O}_{z, \varepsilon}}\left|\nabla w_{0}^{N}\right|^{2} d x=\sum_{q=0}^{m-3} \varepsilon^{q+3} \mathcal{K}_{q}^{3, N}(z, \mathcal{O})+o\left(\varepsilon^{m}\right),
$$


KOHN-VOGELIUS FORMULATION AND HIGH-ORDER TOPOLOGICAL ASYMPTOTIC FORMULA FOR IDENTIFYING SMALL OBSTACLES IN A

where $\left\{z \longmapsto \mathcal{K}_{q}^{3, N}(z, \mathcal{O}), 0 \leq q \leq m-3\right\}$ is a set of functions, defined in $\Omega$ by

$$
\mathcal{K}_{q}^{3, N}(z, \mathcal{O})=\sum_{p=0}^{q} \frac{\nu}{p !(q-p) !} \int_{\mathcal{O}} D^{(p+1)} w_{0}^{N}(z)\left(y^{p}\right): D^{(q-p+1)} w_{0}^{N}(z)\left(y^{q-p}\right) d y .
$$

Proof: The change of variable $x=z+\varepsilon y$, implies

$$
\int_{\mathcal{O}_{z, \varepsilon}}\left|\nabla w_{0}^{N}\right|^{2} d x=\varepsilon^{3} \int_{\mathcal{O}}\left|\nabla w_{0}^{N}(z+\varepsilon y)\right|^{2} d y .
$$

Since $w_{0}^{N}$ is smooth near $z$, by Taylor's theorem it follows

$$
\nabla w_{0}^{N}(z+\varepsilon y)=\sum_{p=0}^{m} \frac{1}{p !} D^{(p+1)} w_{0}^{N}(z)\left(y^{p}\right)+o\left(\varepsilon^{m}\right) .
$$

By Cauchy product formula, we obtain

$\left|\nabla w_{0}^{N}\right|^{2}(z+\varepsilon y)=\sum_{q=0}^{m} \varepsilon^{q}\left(\sum_{p=0}^{q} \frac{1}{p !(q-p) !} D^{(p+1)} w_{0}^{N}(z)\left(y^{p}\right): D^{(q-p+1)} w_{0}^{N}(z)\left(y^{q-p}\right)\right)+o\left(\varepsilon^{m}\right)$.

\subsection{Estimate of the Dirichlet terms}

In this section, we examine the Dirichlet terms involved in the Kohn-Vogelius functional variation. Based on the asymptotic behavior of the perturbed solution $\left(w_{\varepsilon}^{D}, q_{\varepsilon}^{D}\right)$ with respect to the parameter $\varepsilon$, we establish a high-order asymptotic formula for each term of the function $g^{D}(\varepsilon)$.

Using Theorem 4.3, the function $g^{D}(\varepsilon)$ admits the following estimate

$$
\begin{aligned}
\partial^{D}(\varepsilon)= & \sum_{k=0}^{m-1} \varepsilon^{k+1} \int_{\partial \mathcal{O}} \sigma_{y}\left(E_{k}^{D}, \Pi_{k}^{D}\right)(y) \mathbf{n}(y) \cdot w_{0}^{D}(z+\varepsilon y) d s(y) \\
& +\sum_{k=1}^{m-2} \varepsilon^{k+2} \int_{\partial \mathcal{O}} \sigma\left(V_{k}^{D}, S_{k}^{D}\right)(z+\varepsilon y) \mathbf{n} \cdot w_{0}^{D}(z+\varepsilon y) d s(y) \\
& -\varepsilon^{3} \nu \int_{\mathcal{O}}\left|\nabla w_{0}^{D}(z+\varepsilon y)\right|^{2} d y \cdot+o\left(\varepsilon^{m}\right) .
\end{aligned}
$$

Similar to the Naumann case, we derive the following preliminary lemmas estimating the integral terms in the last equality. 
KOHN-VOGELIUS FORMULATION AND HIGH-ORDER TOPOLOGICAL ASYMPTOTIC FORMULA FOR IDENTIFYING SMALL OBSTACLES IN A FLUID MEDIUM

Lemma 5.5. We have

$$
\sum_{k=0}^{m-1} \varepsilon^{k+1} \int_{\partial \mathcal{O}} \sigma_{y}\left(E_{k}^{D}, \Pi_{k}^{D}\right)(y) \mathbf{n}(y) \cdot w_{0}^{D}(z+\varepsilon y) d s(y)=\sum_{q=0}^{m-1} \varepsilon^{q+1} \mathcal{K}_{q}^{1, D}(z, \mathcal{O})+o\left(\varepsilon^{m}\right),
$$

where the functions $z \longmapsto \mathcal{K}_{q}^{1, D}(z, \mathcal{O}), 0 \leq q \leq m$ are defined in $\Omega$ by

$$
\mathcal{K}_{q}^{1, D}(z, \mathcal{O})=\sum_{p=0}^{q} \frac{1}{p !} \int_{\partial \mathcal{O}} \sigma_{y}\left(E_{q-p}^{D}, \Pi_{q-p}^{D}\right)(y) \mathbf{n}(y) \cdot\left[\nabla^{(p)} w_{0}^{D}(z)\left(y^{p}\right)\right] d s(y)
$$

Lemma 5.6. The second integral term in the right hand side of (24) satisfies

$\sum_{k=1}^{m-2} \varepsilon^{k+2} \int_{\partial \mathcal{O}} \sigma\left(V_{k}^{D}, S_{k}^{D}\right)(z+\varepsilon y) \mathbf{n} . w_{0}^{D}(z+\varepsilon y) d s(y)=\sum_{q=1}^{m-2} \varepsilon^{q+2} \mathcal{K}_{q}^{2, D}(z, \mathcal{O})+o\left(\varepsilon^{m}\right)$,

where the functions $z \longmapsto \mathcal{K}_{q}^{2, D}(z, \mathcal{O}), 1 \leq q \leq m-2$ are defined in $\Omega$ by

$\mathcal{K}_{q}^{2, D}(z, \mathcal{O})=\sum_{p=0}^{q-1} \sum_{l=0}^{p} \frac{1}{l !(p-l) !} \int_{\partial \mathcal{O}} \sigma^{(l)}\left(V_{q-p}^{D}, S_{q-p}^{D}\right)(z) \mathbf{n} \cdot \nabla^{(p-l)} w_{0}^{D}(z)\left(y^{p-l}\right) d s(y)$

Lemma 5.7. The second term in (22) admits the estimate

$$
\nu \int_{\mathcal{O}}\left|\nabla w_{0}^{D}(z+\varepsilon y)\right|^{2} d y=\sum_{q=0}^{m} \varepsilon^{q} \mathcal{K}_{q}^{3, D}(z, \mathcal{O})+o\left(\varepsilon^{m}\right),
$$

where $\left\{z \longmapsto \mathcal{K}_{q}^{3, D}(z, \mathcal{O}), 0 \leq q \leq m\right\}$ is a set of functions, defined in $\Omega$ by

$$
\mathcal{K}_{q}^{3, D}(z, \mathcal{O})=\sum_{p=0}^{q} \frac{1}{p !(q-p) !} \int_{\mathcal{O}} \nabla^{(p+1)} w_{0}^{D}(z)\left(y^{p}\right): \nabla^{(q-p+1)} w_{0}^{D}(z)\left(y^{q-p}\right) d y .
$$

\subsection{Topological asymptotic formula}

In this section, we derive a high-order topological asymptotic expansion for the Kohn-Vogelius functional $\mathcal{K}$. The main result of this section is described by Theorem 5.8 .

Theorem 5.8. Let $\mathcal{O}_{z, \varepsilon}=z+\varepsilon \mathcal{O}$ be a small obstacle inserted in the fluid flow domain $\Omega$. In the presence of $\mathcal{O}_{z, \varepsilon}$, the Kohn-Vogelius functional $\mathcal{K}$ satisfies the following high-order asymptotic expansion

$$
\mathcal{K}\left(\Omega \backslash \overline{\mathcal{O}_{z, \varepsilon}}\right)=\mathcal{K}(\Omega)+\sum_{q=1}^{m} \varepsilon^{q} \delta^{q} \mathcal{K}(z, \mathcal{O})+o\left(\varepsilon^{m}\right)
$$


KOHN-VOGELIUS FORMULATION AND HIGH-ORDER TOPOLOGICAL ASYMPTOTIC FORMULA FOR IDENTIFYING SMALL OBSTACLES IN A

where $\delta^{q} \mathcal{K}$ is the $q^{\text {th }}$ topological derivative order, defined in $\Omega$ by:

- If $q=1,2$

$$
\delta^{q} \mathcal{K}(z, \mathcal{O})=\left[\mathcal{K}_{q-1}^{1, N}-\mathcal{K}_{q-1}^{1, D}\right](z, \mathcal{O})
$$

- If $3 \leq q \leq m$

$\delta^{q} \mathcal{K}(z, \mathcal{O})=\left[\mathcal{K}_{q-1}^{1, N}-\mathcal{K}_{q-1}^{1, D}\right](z, \mathcal{O})+\left[\mathcal{K}_{q-2}^{2, N}-\mathcal{K}_{q-2}^{2, D}\right](z, \mathcal{O})-\left[\mathcal{K}_{q-3}^{3, N}-\mathcal{K}_{q-3}^{3, D}\right](z, \mathcal{O})$.

Proof. According to Lemmas 5.1, 5.2, 5.3 and 5.4, the Neuman term $g^{N}(\varepsilon)$ satisfies the estimate

$\mathcal{J}^{N}(\varepsilon)=\sum_{q=0}^{m-1} \varepsilon^{q+1} \mathcal{K}_{q}^{1, N}(z, \mathcal{O})+\sum_{q=1}^{m-2} \varepsilon^{q+2} \mathcal{K}_{q}^{2, N}(z, \mathcal{O})-\sum_{q=0}^{m-3} \varepsilon^{q+3} \mathcal{K}_{q}^{3, N}(z, \mathcal{O})+o\left(\varepsilon^{m}\right)$.

Based on Lemmas 5.5, 5.6 and 5.7, the Dirichlet term $\mathcal{J}^{D}(\varepsilon)$ can be estimated as

$\mathcal{J}^{D}(\varepsilon)=\sum_{q=0}^{m-1} \varepsilon^{q+1} \mathcal{K}_{q}^{1, D}(z, \mathcal{O})+\sum_{q=1}^{m-2} \varepsilon^{q+2} \mathcal{K}_{q}^{2, D}(z, \mathcal{O})-\sum_{q=0}^{m-3} \varepsilon^{q+3} \mathcal{K}_{q}^{3, D}(z, \mathcal{O})+o\left(\varepsilon^{m}\right)$.

Combining the two previous estimates and using the fact that

$$
\mathcal{K}\left(\Omega \backslash \overline{\mathcal{O}_{z, \varepsilon}}\right)-\mathcal{K}(\Omega)=J^{N}(\varepsilon)-\mathcal{J}^{D}(\varepsilon),
$$

one can derive the desired asymptotic formula.

\section{Conclusion}

In this paper, we have derived a high-order topological asymptotic formula describing the variation of the Kohn-Vogelius functional with respect to the presence of a small obstacle immersed in the fluid flow domain. The obtained formula can serve as very useful tools for the numerical identification of the location " $z$ ", the size " $\varepsilon$ " and the shape " $O$ " of the unknown obstacle.

From the asymptotic formula in Theorem 5.8, it now follows that, up to terms of smaller order, the unknown parameters $z, \varepsilon$ and $\mathcal{O}$ can be characterized as the solution of a parameters estimate problem minimizing the nonlinear scalar function

$$
\mathcal{F}(\varepsilon, z, \mathcal{O})=\sum_{q=1}^{m} \varepsilon^{q} \delta^{q} \mathcal{K}(z, \mathcal{O}) .
$$


KOHN-VOGELIUS FORMULATION AND HIGH-ORDER TOPOLOGICAL ASYMPTOTIC FORMULA FOR IDENTIFYING SMALL OBSTACLES IN A FLUID MEDIUM

A first task of the identification process, is then the determination of the location " $z$ " (center of the obstacle) and the size " $\varepsilon$ ". A second task would be (as well as possible) the determination of other information about the obstacle, such as its shape "O".

A detailed account of this work and some numerical investigations will be the subject of a forthcoming paper.

\section{References}

[1] G. Alessandrini and A. Diaz Valenzuela, Unique determination of multiple cracks by two measurements, SIAM. J. Control Optim. 34 (3), 1996, 913921.

[2] G. Alessandrini, E. Beretta and S. Vessela Determining linear cracks by boundary measurements: Lipschitz stability, SIAM J. Math. Anal. 27, 1996, 361-375.

[3] S. Andrieux and A. Ben Abda Identification of planar cracks by complete overdetermined data: inversion formulae, Inverse Problems, 12, 1996, 553-563.

[4] A. Ben Abda, H. Ben Ameur and M. Jaoua Identification of $2 D$ cracks by elastic boundary measurements, Inverse Problems 15, 1999, 67-77.

[5] N. Nishimura and S. Kobayashi A boundary integral equation method for an inverse problem related to crack detection, Int. J. Num. Methods Eng., 32(1991), pp. 1371-1387.

[6] H. Ammari, S. Moskow, and M. Vogelius, Boundary integral formulas for reconstruction of electromagnetic imperfections of small diameter, ESAIM, Cont. Opt. Cal. Variat. vol. 9, 2004, 49-66.

[7] M. Vogelius, and D. Volkov, Asymptotic formulas for perturbations in the electromagnetic fields due to the presence of inhomogeneities, Math. Model. Numer. Anal. 34 (2000), 723-748.

[8] E. Beretta, E. Francini, and M. Vogelius, Asymptotic formulas for for steady state voltage potentials in the presence of thin inhomogeneities. A rigorous error analysis, J. Math. Pures Appl. 82, 2003, 1277-1301.

[9] D.J. Cedio-Fengya, S. Moskow, and M. Vogelius, Identification of conductivity imperfections of small diameter by boundary measurements. Continuous dependence and computational reconstruction, Inverse Problems. vol. 14, 1998, 553-595.

[10] A. Friedman, and M. Vogelius, Identification of small inhomogeneties of extreme conductivity by boundary measurements: a theorem on continuous dependence, Arch. Rat. Mech. Anal., 105, 1989, 299-326. 
KOHN-VOGELIUS FORMULATION AND HIGH-ORDER TOPOLOGICAL ASYMPTOTIC FORMULA FOR IDENTIFYING SMALL OBSTACLES IN A FLUID MEDIUM

[11] C. Alves, and H. Ammari, Boundary integral formulae for the reconstruction of imperfections of small diameter in an elastic medium, SIAM, J. Appl. Math. 62, 2001, 94-106.

[12] H. Ammari, H. Kang, G. Nakamura and K. Tanuma, Complete asymptotic expansions of solutions of the system of elastostatics in the presence of an inclusion of small diameter and detection of an inclusion, J. Elasticity 67, 2002, 97-129.

[13] H. Ammari, M. Vogelius and D. Volkov, Asymptotic formulas for perturbations in the electromagnetic fields due to presence of inhomogeneities of small diameter II. The full Maxwell's equations, J. Math. Pures Appl. 80, 2001, 769-814.

[14] F. Caubet, M. Dambrine, D. Kateb, and C. Z. Timimoun, A KohnVogelius formulation to detect an obstacle immersed in a fluid. Inverse Probl. Imaging, 7(1):123-157, (2013).

[15] S. Andrieux, T.N. Baranger, A. Ben Abda, Solving Cauchy problems by minimizing an energy-like functional Inverse Problems, 22 (2006) 115-134.

[16] S. Garreau, Ph. Guillaume, M. Masmoudi, The topological asymptotic for PDE systems: The elastics case, SIAM J. contr. Optim. 39(6), 1756-1778, (2001).

[17] Ph. Guillaume and K. Sid Idris, The topological asymptotic expansion for the Dirichlet Problem. SIAM J. Control. Optim. 41, 1052-1072, (2002).

[18] Ph. Guillaume, K. Sid Idris, Topological sensitivity and shape optimization for the Stokes equations, SIAM J. Control Optim. 43 (1) 1-31, (2004).

[19] M. Hassine, Shape optimization for the Stokes equations using topological sensitivity analysis. ARIMA, 5:216-229, (2006).

[20] S. Amstutz, The topological asymptotic for the Navier-Stokes equations. ESAIM Control Optim. Calc. Var., 11(3):401-425 (electronic), (2005).

[21] M. Abdelwahed, M. Hassine, Topological optimization method for a geometric control problem in Stokes flow. Appl. Numer. Math. 59(8), 18231838, (2009).

[22] M. Hassine, M. Masmoudi, The topological asymptotic expansion for the quasi-Stokes problem. ESAIM. Control, Optimisation and Calculus of Variations, vol. 10, no. 4, pp. 478-504, 2004.

[23] M. Masmoudi, J. Pommier, and B. Samet, The topological asymptotic expansion for the Maxwell equations and some applications. Inverse Problems, 21(2):547-564, (2005).

[24] S.T. Chung and T.H. Kwon, Numerical simulation of fiber orientation in injection moulding of short-fiber-reinforced thermoplastics, Polym. Eng. Sci. 7 (35), 1995, 604-618. 
KOHN-VOGELIUS FORMULATION AND HIGH-ORDER TOPOLOGICAL ASYMPTOTIC FORMULA FOR IDENTIFYING SMALL OBSTACLES IN A FLUID MEDIUM

[25] X. Fan, N. Phan-Thien and R. Zheng, A direct simulation of fiber suspensions, J. Non-Newtonian Fluid Mech. 74, 1998), 113-135.

[26] T. Gotz, Simulating particles in Stokes flow, J. Comput. Appl. Math. 175 (2005), 415-427.

[27] C. Pozrikidis, Dynamic simulation of the flow of suspensions of twodimensional particles with arbitrary shapes, Eng. Anal. Boundary Elements 25 (1) (2001), pp.19-30.

[28] H. Zhou and C. Pozrikidis, Adaptive singularity method for Stokes flow past particles, J. Comput. Phys. 117 (1995), pp. 79-89.

[29] R. Codina, U. Schaffer and E. Oñate, Mould filling simulation using finite elements, Int. J. Numer. Meth. Fluid Flow 4, 1994, 291-310.

[30] G. Dhatt, D.M. Gao, A. Ben Cheikh, A finite element simulation of metal flow in moulds, Int. J. Numer. Meth. Eng. 30, 1990, 821-831.

[31] K.M. Shyue, A fluid-mixture type algorithm for compressible multicomponent flow with van der Waals equation of state, J. Comput. Phys. 156 (1999), pp. 43-88.

[32] A. Ben Abda, M. Hassine, M. Jaoua, M. Masmoudi, Topological sensitivity analysis for the location of small cavities in Stokes flow,SIAM J. Contr. Optim , 2871-2900, (2009).

[33] F. Caubet, M. Dambrine, Localization of small obstacles in Stokes flow. Inverse Problems, 28(10):105007, 31, (2012).

[34] L. Afraites, M. Dambrine, K. Eppler AND K.Kateb, Detecting perfectly insulated obstacles by shape optimization techniques of order two. ,Discrete Contin.Dyn. Syst. , no 8(2), 389-416, (2007).

Montassar Barhoumi,

Ecole Supérieure des Sciences et de la Technologie de Hammam Sousse,

Sousse University, Sousse University.

Email: montassar.barhoumi2014@gmail.com 